\title{
Outcomes of Non-Alcoholic Steatohepatitis Patients After Liver Transplantation: A Systematic Review and Meta-Analysis
}

\author{
Yu Zhao ${ }^{1}$, Xiaoguang Zhan ${ }^{1}$ and Yan Zhang ${ }^{1, *}$ \\ ${ }^{1}$ Surgery Department, Tianjin Second People's Hospital, Tianjin, China \\ "Corresponding author: Surgery Department, Tianjin Second People’s Hospital, Tianjin, China. Email: adamadm@sina.com
}

Received 2018 July 18; Revised 2019 March 25; Accepted 2019 April 02.

\begin{abstract}
Context: Non-alcoholic steatohepatitis (NASH) has become a prevalent disease worldwide as well as a leading cause for liver transplantation in the next decades. Thus the purpose of this review is to evaluate the outcomes of NASH patients after liver transplantation in comparison to non-NASH patients.

Evidence Acquisition: Studies published in English before February 2018 were retrieved through literature searches in PubMed, Web of Science, Wiley Online Library, Research Gate, and EMBASE. Studies that compared the outcomes of liver transplantation for NASH and other liver diseases were eligible.

Results: A total of 13 studies were included in the current study. Compared with non-NASH group, NASH patients presented higher mortality after liver transplantation in 1 and 2 years, which might associate with recipient age, sex, diabetes, and hypertension. However, the 3- and 5-year survival were similar between the two groups. Infection was the most prevalent etiology of mortality in NASH group.

Conclusions: The NASH patients receiving liver transplantation presented higher mortality in the first two years and infectious complications were fatal for them. Therefore, more attention should be paid on NASH patients with infections, especially in the first and second year after the transplantation.
\end{abstract}

Keywords: Non-Alcoholic Steatohepatitis, Non-Alcoholic Fatty Liver Disease, Liver Transplantation, Meta-Analysis

\section{Context}

Non-alcoholic fatty liver disease (NAFLD) is a worldwide disease affecting up to $45 \%$ of adults and $10 \%$ of children in western countries $(1,2)$. Several studies also showed an increase occurred in eastern Asia owing to the shift in lifestyle and dietary patterns $(3,4)$. The NAFLD represents a scope of clinical and pathologic conditions featured by macro-vesicular steatosis in the absence of alcohol, which is comprised of a wide gamut of liver diseases such as nonalcoholic steatohepatitis (NASH), fibrosis, cirrhosis, and hepatocellular carcinoma.

The NASH is a progressive form of NAFLD that can progress to cirrhosis or liver failure, requiring liver transplantation (LT) (5), which is also served as a reason for the surgery in the next decades (6). Although LT has become a standard treatment for acute and chronic end-stage liver disease contributed by NASH, there is not a consensus on the outcomes of the operation. Several separate studies (711) have shown that LT for NASH had increased mortality in early months or years compared to those treated for other indications, while no difference could be observed regarding long-term survival. Moreover, the reason for the shortterm mortality is still unclear.

Therefore, in the current meta-analysis and systematic review, first, we aimed to compare the survival between the NASH- and non-NASH patients after LT and figure out the potential factors associated with mortality at specific time point; second, to compare the causes for death after LT.

\section{Evidence Acquisition}

This article follows the PRISMA guidelines for reporting meta-analysis and systematic review.

\subsection{Data Sources and Searches}

Studies published in English before February 2018 were surveyed through online databases PubMed, EMBASE, Web of Science, Wiley Online Library, and Research Gate. Search terms consisted of the following keywords: "Non-alcoholic steatohepatitis" OR "NASH" OR "Non-alcoholic fatty liver disease" OR “NAFLD” AND "liver transplantation". All titles 
and abstracts from the initial search were screened by two reviewers. If the title and abstracts did not contain enough information to include or exclude the study from the analysis, the study was reviewed in full-text. Citation lists of relevant articles and reviews were additionally scanned to identify further studies of interest.

\subsection{Eligibility Criteria}

Studies conforming to the following selection criteria were included in the meta-analysis: first, focus on adult patients; second, compare the outcomes after liver transplantation between NASH and non-NASH patients; and third, contain available data related to patient survival according to time spectrum and reasons for mortality. For relevant studies that did not provide necessary data for analysis, we contacted the corresponding author of the articles for information. If we did not receive the author's response in a reasonable amount of time, the study was excluded from the meta-analysis.

\subsection{Data Extraction}

All retrieved records from separate studies were reviewed and extracted by using standardized forms. Discrepancies were resolved by referring to the original articles and discussing by all authors. Following details were extracted from each study: first author, year of publication, sample size, patient baseline characteristics, survival, and causes of death.

\subsection{Quality Assessment and Risk of Bias}

According to the Cochrane Handbook of Systematic Review of Interventions, cohort control studies were assessed by the Newcastle-Ottawa Scale (NOS) based on three perspectives: selection, comparability, and outcome of the cohort. The NOS score ranged from 0 to 9, and studies with a total score of 6 or lower were considered low quality, while studies with a total score of 7 or above were regarded as high quality and eligible for inclusion in the meta-analysis. Details are presented in Table 1.

\subsection{Statistical Analysis}

All statistical analyses were performed by Review Manager version 5.3. Results and effect size analysis were presented as odds ratio (OR) and numbers were presented with $95 \%$ confidence intervals (95\% CI).

The $\mathrm{I}^{2}$ statistics, chi-squared test, and $95 \% \mathrm{CI}$ were performed to assess heterogeneity. The $\mathrm{I}^{2}$ describes the percentage of the variability in effect estimates that is due to heterogeneity rather than sampling error (chance). An $\mathrm{I}^{2}$ value $>50 \%$ or P value $<0.05$ was considered substantial heterogeneity, and the random effect model was selected, otherwise, the fixed effect model was employed. Sensitivity analysis was conducted to confirm the robustness of the results by omitting one study at a time. Publication bias was assessed by the use of the funnel plot.

In order to deliver a more accurate result and figure out the factors related to mortality, subgroup analysis was performed. The subgroup was categorized based on five significant factors: recipient age ( $\leq 58$ or $>58$ ), female patient ( $\leq 40 \%$ or $>40 \%$ ), MELD score $(\leq 20$ or $>20$ ), diabetes proportion $(\leq 60 \%$ or $>60 \%)$, and hypertensive proportion $(\leq$ $50 \%$ or $>50 \%)$.

\section{Results}

\subsection{Search Process}

Detailed screening processes were shown in Figure 1. A total of 1459 articles were initially selected from PubMed Web of Science, Wiley Online Library, Research Gate, and EMBASE, and 9 from a manual search of citation list. Out of 987 manuscripts after removing duplicates, 538 were excluded due to inappropriate article styles such as review, case report, comment, etc. Out of 449 manuscripts following title and abstract screening, 401 were excluded due to low relativity. Out of 48 articles fully assessed, 35 were excluded due to the paucity of the detailed description on the surgery or patient characteristics or essential endpoints and finally, 13 were included for meta-analysis.

\subsection{Characteristics of Enrolled Studies}

Table 2 shows the basic information of the enrolled studies in this meta-analysis. The thirteen enrolled trials based on a total of 4806 participants were published in English from 2009 to 2017, which were all retrospective cohort study. The control group was composed of the patients with alcoholic liver disease, alcoholic cirrhosis, and hepatitis $C$ virus required liver transplantation. A large number of the patients were Caucasian with age up to 50 . Diabetes and hypertension were frequently mentioned in the participants except for two studies without any reference for these diseases. In terms of risk of bias, the NOS scores of all included studies are $\geq 6$ points, indicating a lower risk of bias.

\subsection{Patient Survival}

Ten studies reported 1-year survival, four reported 2year survival, eight reported 3-year survival, and eleven reported 5-year survival. The pooled results are presented in Figure 2.

Short-term survival of 1- and 2-year favored the nonNASH group with summary ORs of 0.74 (95\% CI, $0.59-0.93$ ) 


\begin{tabular}{|c|c|c|c|c|c|c|c|c|c|c|}
\hline Study & A & в & c & D & $\mathbf{E}$ & $\mathbf{F}$ & G & H & I & Total Score \\
\hline Agopian et al., 2012 (12) & Yes & Yes & Yes & Yes & No & No & Yes & Yes & Yes & 7 \\
\hline Barritt et al., 2011 (9) & Yes & Yes & Yes & Yes & No & No & Yes & Yes & Yes & 7 \\
\hline Bhagat et al., 2009 (13) & Yes & Yes & Yes & Yes & No & No & Yes & Yes & Yes & 7 \\
\hline Houlihan, 2011 & Yes & Yes & Yes & Yes & No & No & Yes & Yes & Yes & 7 \\
\hline Kennedy et al., 2012 (10) & Yes & Yes & Yes & Yes & No & No & Yes & Yes & Yes & 7 \\
\hline Malik et al., 2009 (7) & Yes & Yes & Yes & Yes & No & No & Yes & Yes & Yes & 7 \\
\hline Park, 2009 (3) & Yes & Yes & Yes & Yes & No & No & Yes & Yes & No & 6 \\
\hline Reddy et al., 2012 (14) & Yes & Yes & Yes & Yes & No & No & No & Yes & Yes & 6 \\
\hline Sourianarayanane et al., 2017 (11) & Yes & Yes & Yes & Yes & Yes & Yes & Yes & Yes & Yes & 9 \\
\hline Than, 2017 & Yes & Yes & No & Yes & No & Yes & Yes & Yes & Yes & 7 \\
\hline Unger, 2016 & Yes & Yes & No & Yes & Yes & Yes & Yes & Yes & Yes & 8 \\
\hline Vanwagner et al., 2012 (15) & Yes & Yes & Yes & Yes & Yes & No & Yes & Yes & Yes & 8 \\
\hline Pizza, 2016 & Yes & Yes & Yes & Yes & Yes & Yes & No & Yes & Yes & 8 \\
\hline
\end{tabular}

and 0.71 (95\% CI, 0.51 - 0.99), respectively. Low heterogeneity can be observed $\left(\mathrm{I}^{2}=15 \%, \mathrm{P}=0.30 ; \mathrm{I}^{2}=0 \%, \mathrm{P}=0.83\right)$. However, there is no significant difference regarding long-term survival of 3- and 5- year with summary ORs of 0.94 (95\% CI, 0.76 - 1.15) and 0.85 (95\% CI, 0.63 - 1.14). Significant heterogeneity can be observed in 5 - year survival $\left(\mathrm{I}^{2}=52 \%, \mathrm{P}=\right.$ $0.02)$.

\subsection{Causes for Death}

Five causes for death were frequently reported, eight studies on infection, seven on cardiac events, five on graft failure, four on malignancy, and four on biliary or operative complications. Detailed pooled results are presented in Figure 3.

More NASH patients died for infection with a summary OR of 1.80 (95\% CI, 1.25 - 2.59), while the non-NASHs were susceptible to graft failure and malignancies with summary ORs of 0.25 (95\% CI, 0.07 - 0.88) and 0.38 (95\% CI, 0.15 $0.97)$, respectively. There are no differences in terms of cardiac events and biliary/operative complications between the two groups with summary ORs of 1.54 (95\% CI, 0.95 2.50) and 0.41(95\% CI, 0.11-1.49). No significant heterogeneity can be found in these five categories.

\subsection{Subgroup and Sensitivity Analyses}

To figure out the factors causing short-term mortality in NASH group, subgroup analysis was performed by categorizing the results into five categories: recipient age $(\leq 58$ or $>58$ ), female percentage ( $\leq 40 \%$ or $>40 \%$ ), MELD score ( $\leq 20$ or $>20$ ), diabetes percentage ( $\leq 60 \%$ or $>60 \%$ ), and hypertension percentage ( $\leq 50 \%$ or $>50 \%$ ).

All subgroup results were consistent with the main outcomes, except for the subgroup of recipient age $>58$, female percentage $>40 \%$, diabetes percentage $>60 \%$ and hy- pertension percentage $>50 \%$, in which the 1-year survival favored non-NASH patients, with summary ORs of 0.61 (95\% CI, 0.43 - 0.85), 0.75 (95\% CI, 0.59 - 0.93), 0.61 (95\% CI, 0.43 0.88 ) and 0.58 (95\% CI, 0.39 - 0.85), respectively. Details are presented in Table 3.

Sensitivity analysis was performed on the results of 5year survival, which presented substantial heterogeneity. When omitted the data from Agopian et al., the heterogeneity was decreased $\left(\mathrm{I}^{2}=0 \%, \mathrm{P}=0.58\right)$ and the pooled results indicated that the non-NASH group presented significantly higher survival rate compared with the NASH group in the 5-year follow-up, which was different from the overall results.

\subsection{Publication Bias}

Since publication bias analysis is significant for the pooled outcomes based on $\geq 10$ articles, only 1-and 5-year survival were subjected to the publication bias assessment in the current study. The results revealed low publication bias and were presented in Figure 4.

\section{Discussion}

In this meta-analysis, pooled statistics of 13 studies indicated that firstly, NASH patients had unfavorable survival in 1 and 2 years after LT compared with non-NASH patients, while there is no significant difference in long-term survival of 3 and 5 years; secondly, infection was the most common etiology of mortality in NASH group, whereas non-NASH was more susceptible to graft failure and malignancy; thirdly, recipient age, sex, diabetes, and hypertension were associated with the short-term morality in NASH patients. 
PRISMA 2009 Flow Diagram

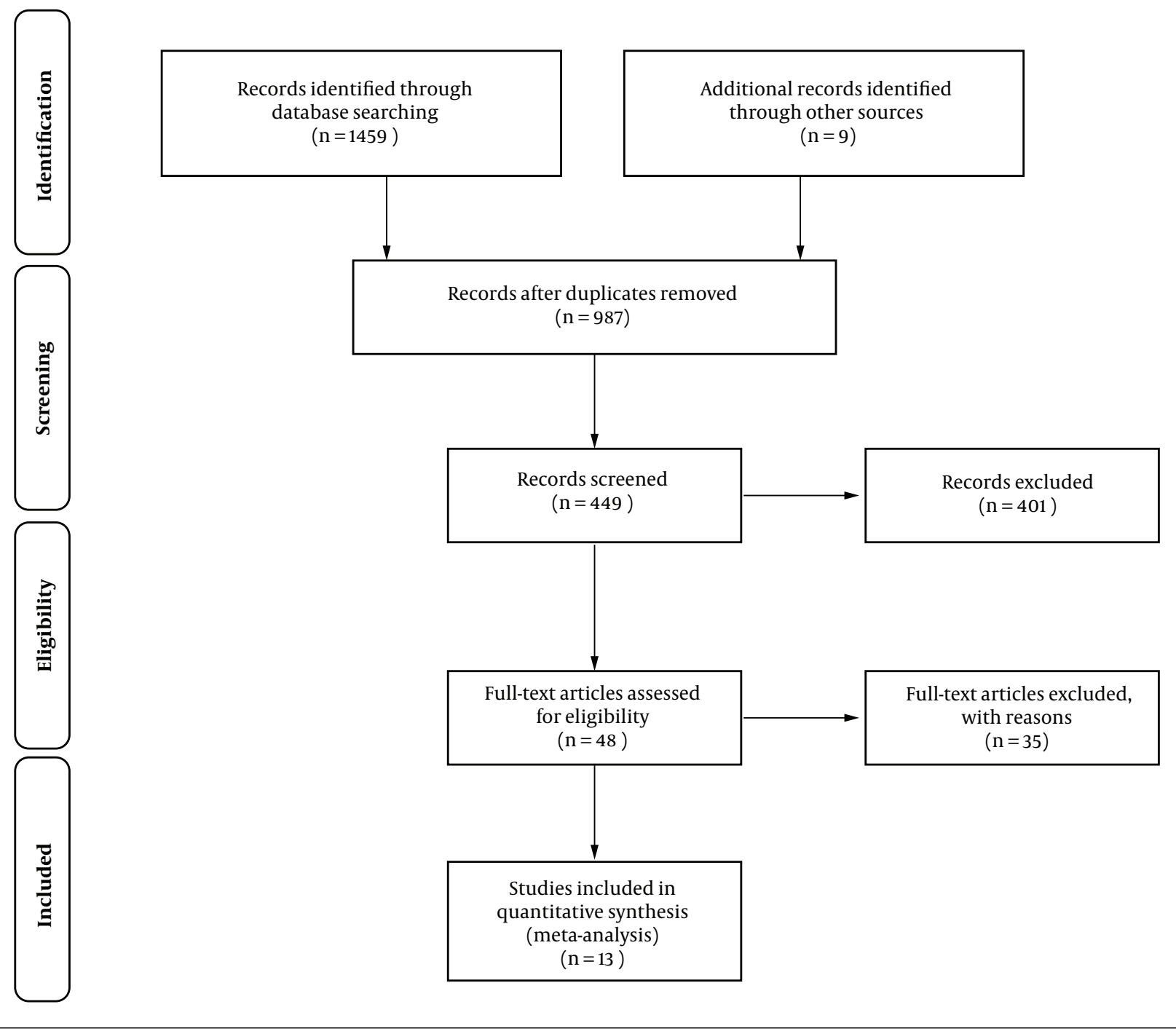

Figure 1. Flow chart of the study selection process

As far as we know, there is still no consensus on the survival of NASH patients after LT in the medical community. Some studies observed that NASH patients performed worse in the first one or two years after LT, while some detected that both NASH and non-NASH patients presented similar survival. A meta-analysis (16) on studies published before 2012 concluded that the survival after LT was similar in 1,3 , and 5 years between NASH and non-NASH patients. Three studies (17-19) on the database for liver transplantation also revealed a similar survival during 5 years between the two patient groups. In the current analysis, the pooled results of survival in 3 and 5 years between groups were consistent with these studies, while the NASH patients had higher mortality in the first two years compared with the controls.

In order to figure out the reasons for the high mortality in 1 - 2 years and further confirm our results, a subgroup analysis was carried out. When the results were restricted to recipient age $>58$, female percentage $>40 \%$, diabetes percentage $>60 \%$, and hypertension percentage $>50 \%$, NASH group outcome was worse in 1- year survival. We found that among all enrolled studies, NASH recipients were always older than non-NASH counterparts about two to five years, which was consistent with a previous 


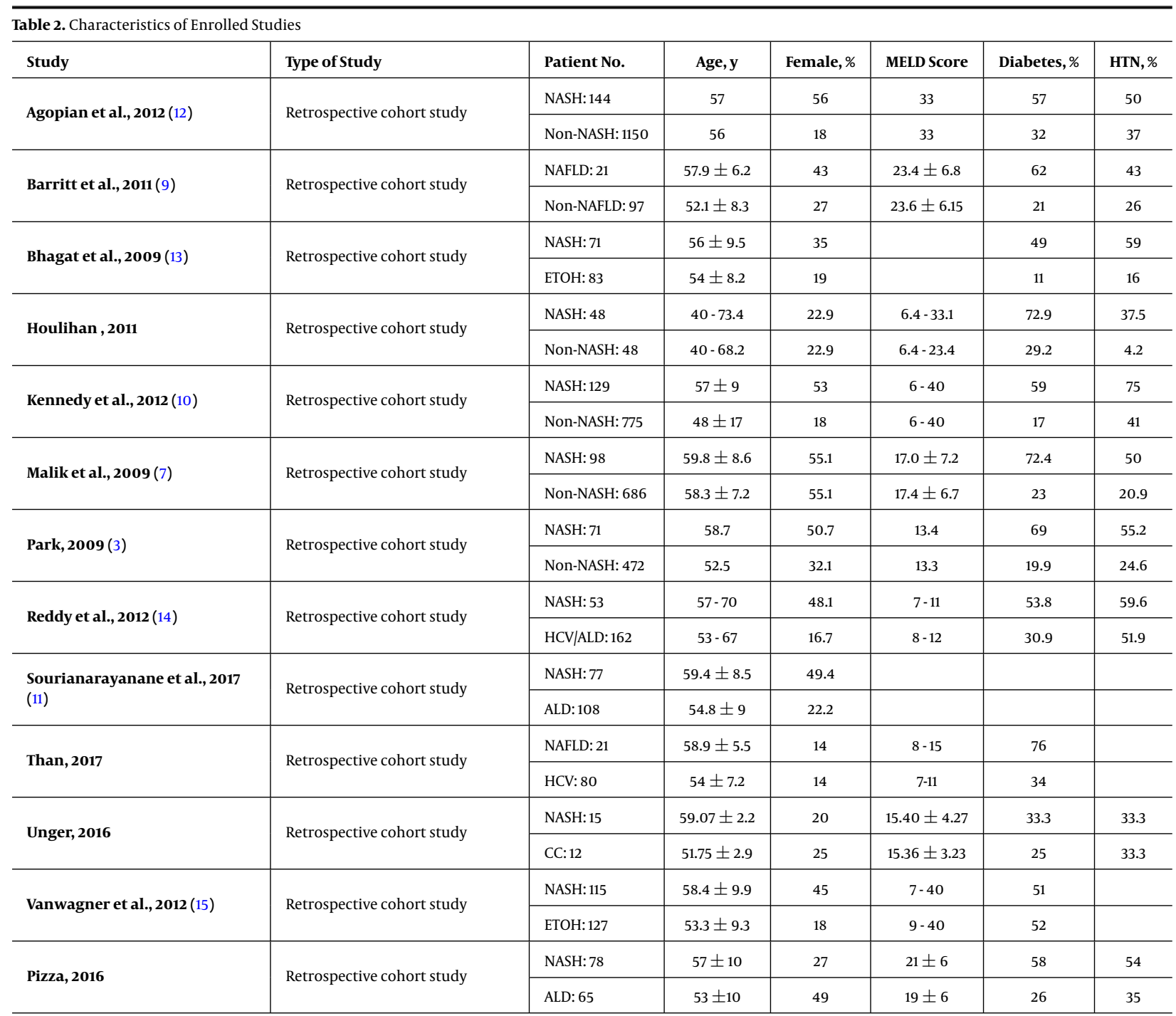

Abbreviations: ALD, alcoholic liver disease; CC, cryptogenic cirrhosis; ETOH, alcoholic cirrhosis; HCV, hepatitis C virus; HTN, hypertension; NAFLD, non-alcoholic fatty liver disease; NASH, non-alcoholic steatohepatitis.

meta-analysis (16), indicating the role of age in the posttransplant outcomes. Charlton et al. also detected that NASH patients are significantly older and more likely to be female (19). However, rare studies discussed gender's influence on patient survival after LT. In this regard, Bhagat et al. (13) declared that gender had no impact on patient survival in the NASH group. Furthermore, different proportions of male and female in each study hampered us to verify the relation between gender and patient survival in this review; therefore, further investigations are still required. Metabolic syndrome has been considered to be related to post-transplant survival in several studies. Contos et al. observed immediate and 1-year mortality after transplantation, which may possess a close relationship with metabolic syndromes such as higher BMI, serious diabetes, and hypertension (20). Barritt et al. found that patients with diabetes had worse survival from 1 to 3 years, which may be due to the adverse effects of immunosuppressive regimes on the metabolic syndrome $(9,21)$. Lorenz et al. reported that diabetes and hypertension were established risk factors for the patient mortality associated with cardiac events (22). Altogether, these results suggested that age, gender, diabetes, and hypertension may affect shortterm survival in the current study.

Through sensitivity analysis, we observed that the heterogeneity was greatly decreased and the pooled ORs was changed in 5-year survival when excluded the article by Agopian et al. (12). In the NOS assessment, this article re- 


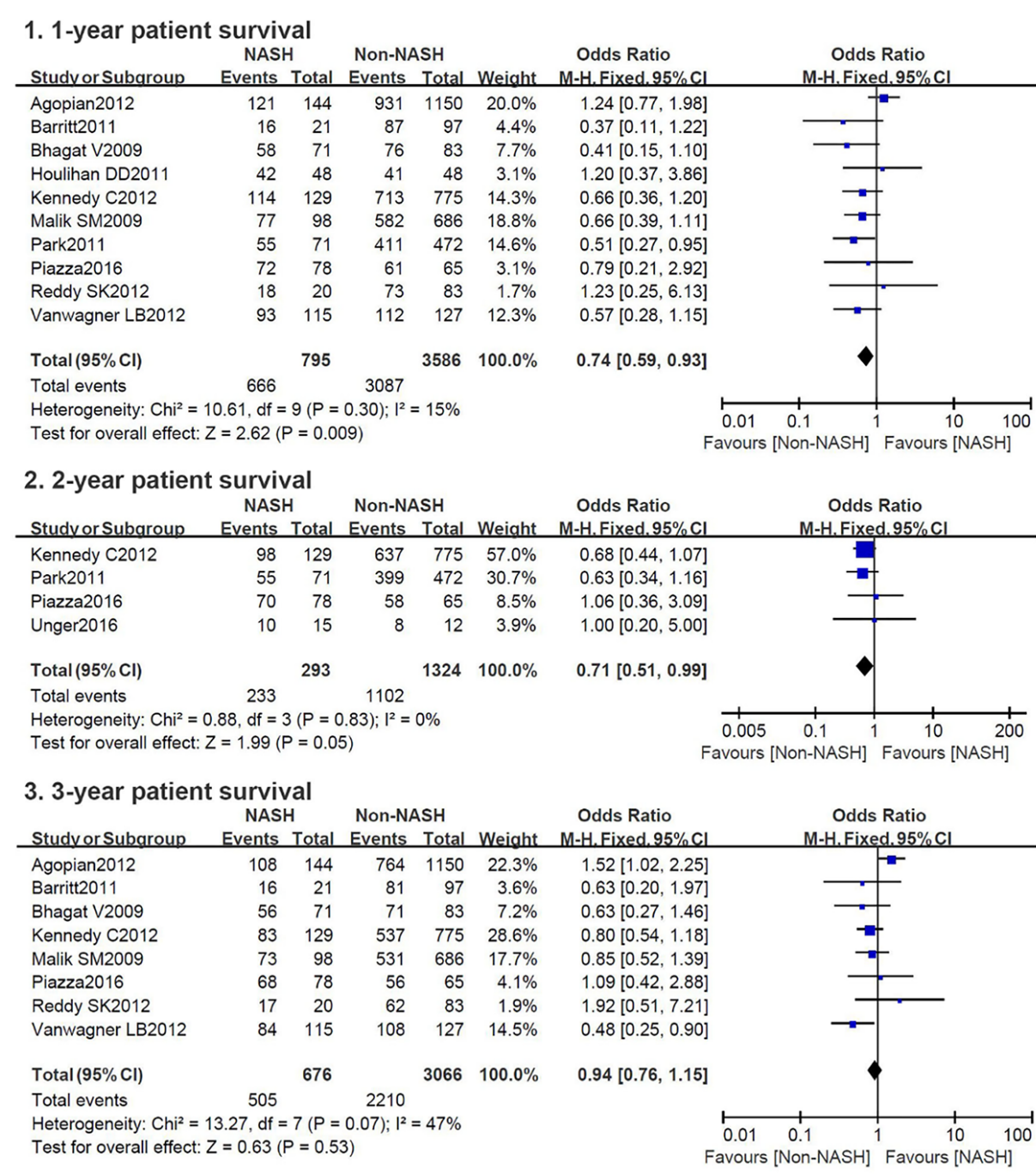

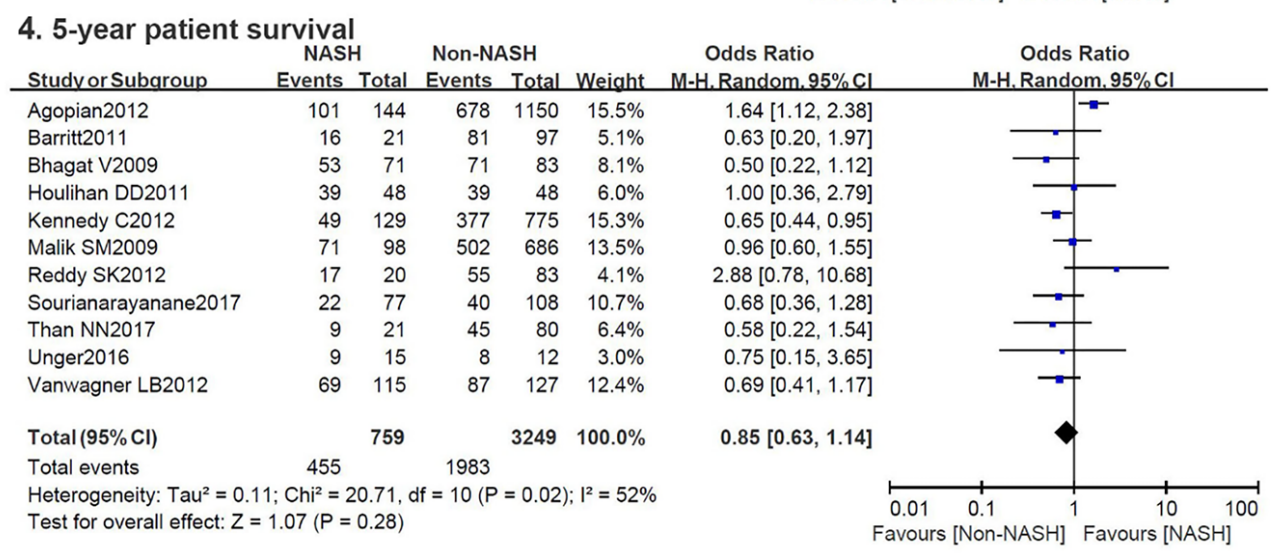

Figure 2. Patient survival at 1-,2-,3- and 5-year after liver transplantation between NASH and non-NASH patients

ceived 7 scores, indicating a low risk of bias and high quality for inclusion, which should not be excluded from the current meta-analysis. Moreover, this article contained a significantly large population accounted for half of the to- 


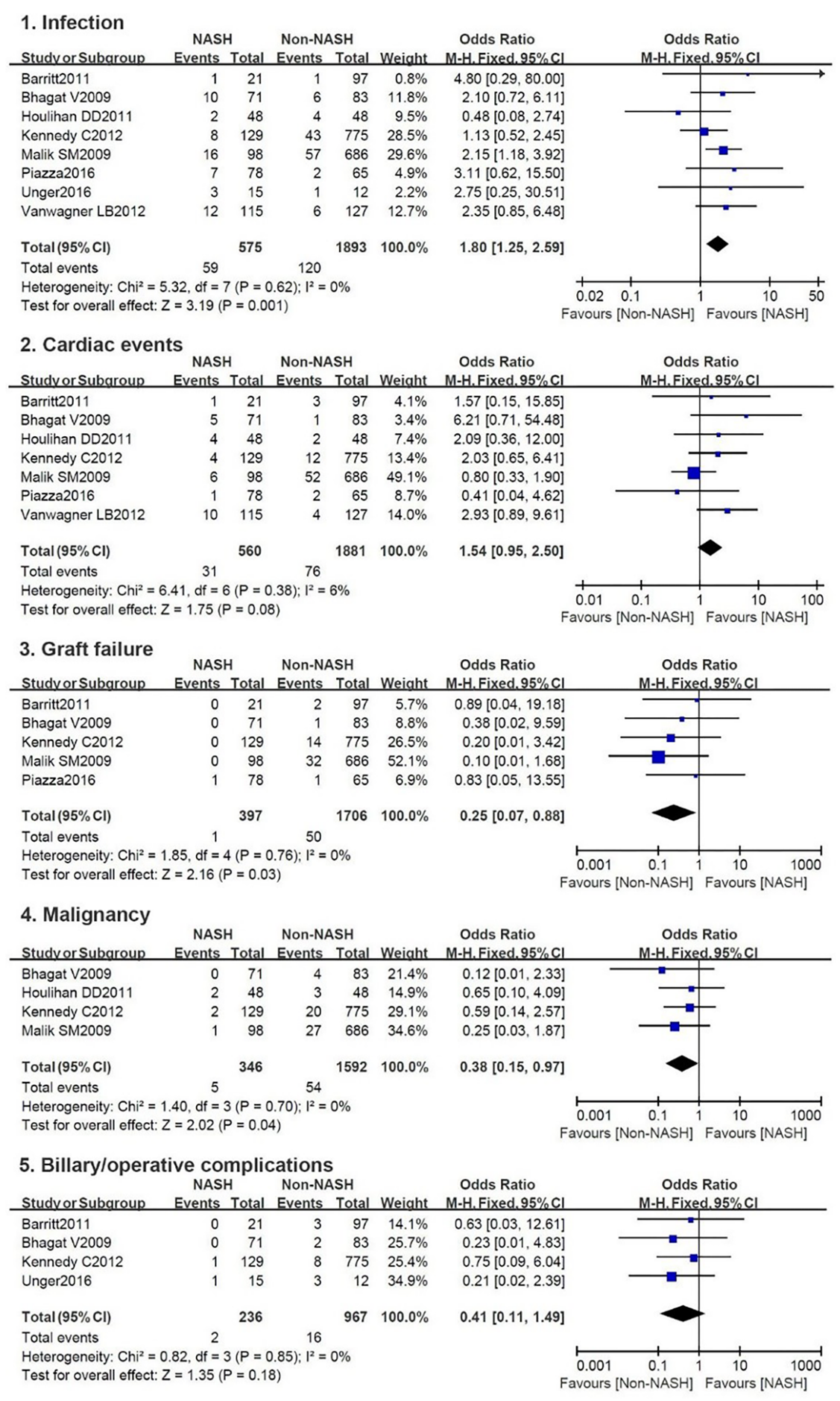

Figure 3. Causes for mortality between NASH and non-NASH patients

tal participant for 5-year survival analysis, indicating its great influence on the overall results. Furthermore, NASH patients had 5-year survival compared with the non-NASH patients and were even significantly better than the pa- 


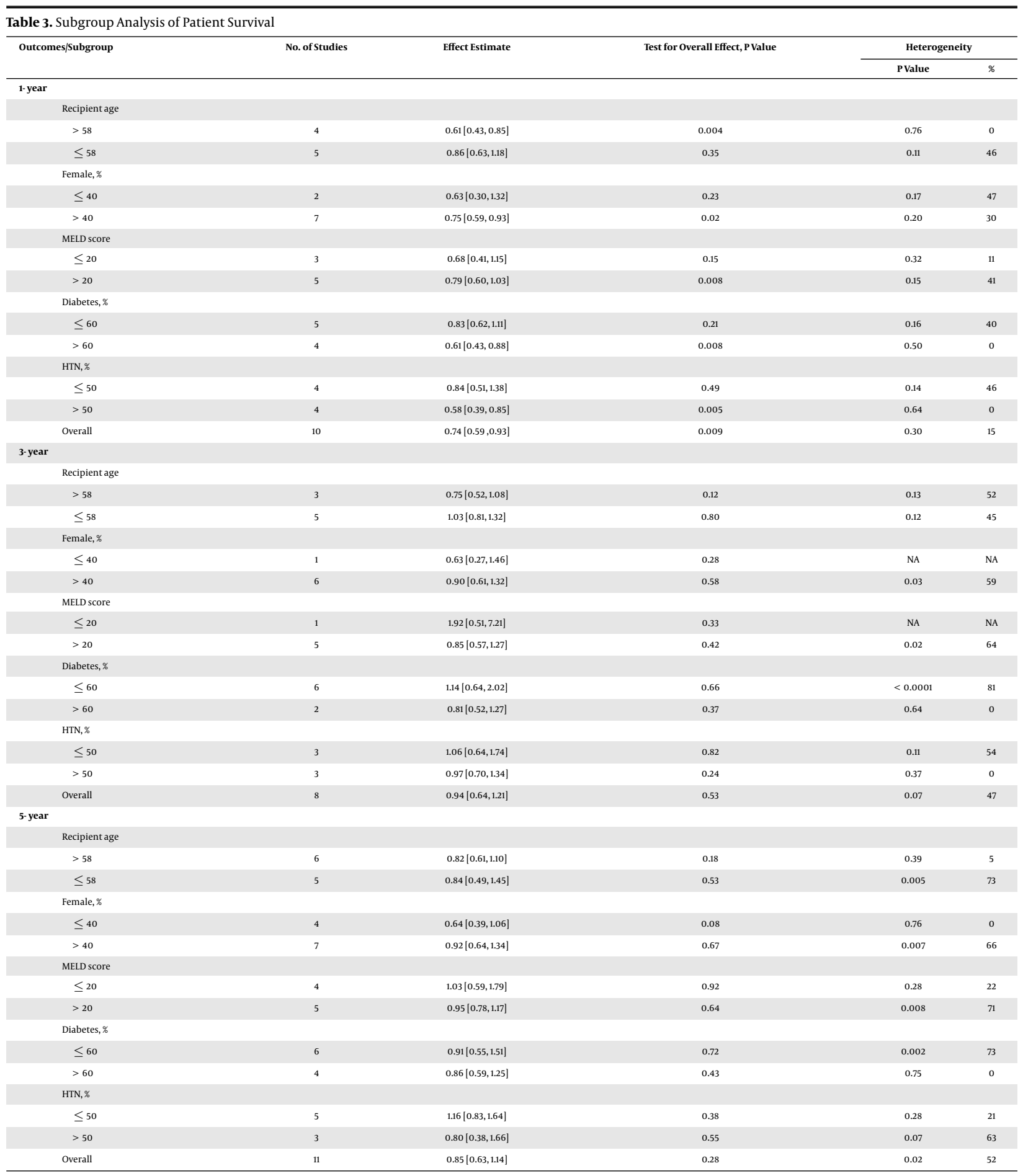

tients with $\mathrm{HCV}$ who represented the largest population in the non-NASH group. However, several studies for pooling yielded a converse result that the 5-year survival more preferred non-NASH group, which might lead to the high heterogeneity. Two previous studies with much larger sam- ple size also analyzed the survival after liver transplantation between NASH and non-NASH patients. One by Afzali et al. (18) based on a population of 53,738 revealed a much pleasant survival rate in NASH group after LT. Another one (19) with 35,781 participants from SRTR database demon- 

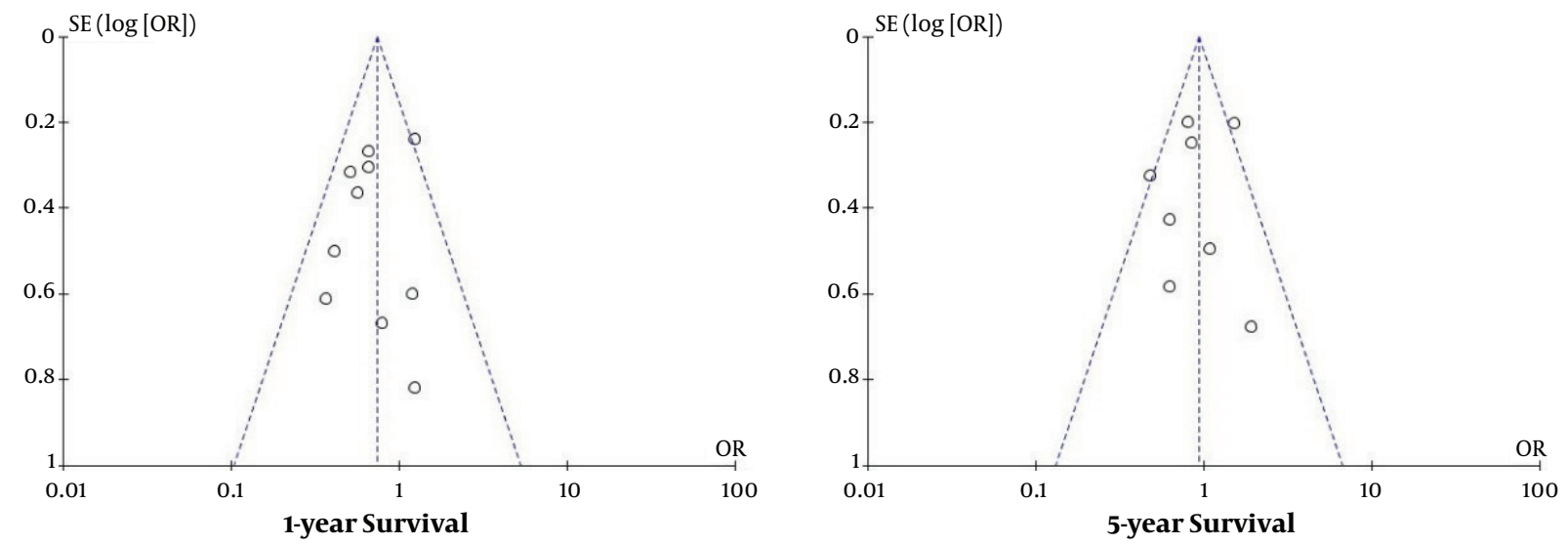

Figure 4. Funnel plot of publication bias

strated that there were no significant differences in posttransplant survival between the two groups. Therefore, we suggested that both NASH and non-NASH presented similar post-transplant outcome in a 5-year follow-up. Moreover, the sample size may be a factor influencing the overall outcomes, and further study based on a larger population is still required.

In the conducted studies, there are several complications accounting for patient mortality after LT such as infection, cardiac events, graft failure, malignancy, biliary or operative complication, etc. It has been reported that infectious complications, including bacterial infections, fungal infections, viral infections, and parasitic infections were responsible for the elevating mortality in the first 3 years after LT, and bloodstream infections featured by sepsis represented the second most common infection (23). Another previous study also observed a significantly higher rate of urogenital tract infection in the NASH group compared with the non-NASH. In this meta-analysis, NASH patients were more susceptible to infections compared with non-NASH patients, which was verified by the above-mentioned studies. Furthermore, several pieces of research also proved that diabetic and hypertensive patients are more inclined to die as a result of infections (13, $14,24)$, and we found that the incidence of diabetes and hypertension are higher in NASH group compared with the non-NASH in this analysis.

Cardiac events have been reported to be the most common cause of mortality in 5 years after LT as well as a relevant cause of death in 10 to 15 years after LT (8). Vanwagner et al. (15) supposed it may be related to attenuated systolic contraction, diastolic relaxation, electrophysiological abnormalities, and the decreasing response of the heart to direct beta stimulation. Montori et al. believed that cardiac events are related to metabolic syndrome(21). Even though more cardiac events can be detected in NASH group, there is still no significant difference between the two groups. Compared with non-NASH group, NASH patients presented lower mortality caused by graft failure and malignancy following liver transplantation. It is reported that NASH group has a lower recurrence of steatosis, which may lead to lower graft failure (13). Also, there are more patients with liver cancer in the non-NASH group, which may explain the high prevalence of malignancy.

There are several limitations to this review should be considered. First, there is no precise definition for NASH in the medical community; therefore, some NASH patients may be ignored in the diagnosis. Second, to avoid patient duplication and low-quality research, we have excluded several studies with large population based on the national database. Third, most included studies did not report the causes for death in a specific period, hampered us to further analyze the complications, which may be responsible for short-term mortality. Fourth, we only included the studies published in English, which may lead to language bias.

In conclusion, liver transplantation is an effective approach for both the NASH and non-NASH patients for longterm benefits, and we suggest that more attention should be paid to NASH patients in the first and second year after liver transplantation, especially those with the following characteristics and symptoms: female, age $>58$, diabetes, hypertension, and post-transplant infections.

\section{Footnotes}

Authors' Contribution: Study concept and design and data extraction: Yu Zhao and Xiaoguang Zhan; literature 
search, data extraction, and analysis: Xiaoguang Zhan and Yu Zhao; data interpretation and manuscript writing: Yu Zhao and Yan Zhang.

Conflict of Interests: There was no conflict of interests in this work.

Funding/Support: The authors declared that the research did not receive any financial grants

\section{References}

1. Schwimmer JB, Deutsch R, Kahen T, Lavine JE, Stanley C, Behling C. Prevalence of fatty liver in children and adolescents. Pediatrics. 2006;118(4):1388-93. doi: 10.1542/peds.2006-1212. [PubMed: 17015527].

2. Williams CD, Stengel J, Asike MI, Torres DM, Shaw J, Contreras M, et al. Prevalence of nonalcoholic fatty liver disease and nonalcoholic steatohepatitis among a largely middle-aged population utilizing ultrasound and liver biopsy: A prospective study. Gastroenterology. 2011;140(1):124-31. doi: 10.1053/j.gastro.2010.09.038. [PubMed: 20858492].

3. Park SH. Current status of liver disease in Korea: Nonalcoholic fatty liver disease. Korean J Hepatol. 2009;15 Suppl 6:S34-9. doi: 10.3350/kjhep.2009.15.S6.S34. [PubMed: 20037278].

4. Li Z, Xue J, Chen P, Chen L, Yan S, Liu L. Prevalence of nonalcoholic fatty liver disease in mainland of China: A meta-analysis of published studies. J Gastroenterol Hepatol. 2014;29(1):42-51. doi: 10.1111/jgh.12428. [PubMed: 24219010].

5. Rafiq N, Bai C, Fang Y, Srishord M, McCullough A, Gramlich T, et al. Long-term follow-up of patients with nonalcoholic fatty liver. Clin Gastroenterol Hepatol. 2009;7(2):234-8. doi: 10.1016/j.cgh.2008.11.005. [PubMed: 19049831].

6. Wang PX, Ji YX, Zhang XJ, Zhao LP, Yan ZZ, Zhang P, et al. Targeting CASP8 and FADD-like apoptosis regulator ameliorates nonalcoholic steatohepatitis in mice and nonhuman primates. Nat Med. 2017;23(4):439-49. doi: 10.1038/nm.4290. [PubMed: 28218919].

7. Malik SM, deVera ME, Fontes P, Shaikh O, Ahmad J. Outcome after liver transplantation for NASH cirrhosis. Am J Transplant. 2009;9(4):782-93. doi:10.1111/j.1600-6143.2009.02590.x. [PubMed: 19344467].

8. Cheong J, Galanko JA, Arora S, Cabezas J, Ndugga NJ, Lucey MR, et al. Reduced impact of renal failure on the outcome of patients with alcoholic liver disease undergoing liver transplantation. Liver Int. 2017;37(2):290-8. doi: 10.1111/liv.13182. [PubMed: 27258535]. [PubMed Central: PMC5136341].

9. Barritt A, Dellon ES, Kozlowski T, Gerber DA, Hayashi PH. The influence of nonalcoholic fatty liver disease and its associated comorbidities on liver transplant outcomes. J Clin Gastroenterol. 2011;45(4):372-8. doi: 10.1097/MCG.0b013e3181eeaffo. [PubMed: 20733515]. [PubMed Central: PMC3292190].

10. Kennedy C, Redden D, Gray S, Eckhoff D, Massoud O, McGuire $\mathrm{B}$, et al. Equivalent survival following liver transplantation in patients with non-alcoholic steatohepatitis compared with patients with other liver diseases. HPB (Oxford). 2012;14(9):625-34. doi: 10.1111/j.1477-2574.2012.00497.x. [PubMed: 22882200]. [PubMed Central: PMC3461389].
11. Sourianarayanane A, Arikapudi S, McCullough AJ, Humar A. Nonalcoholic steatohepatitis recurrence and rate of fibrosis progression following liver transplantation. Eur J Gastroenterol Hepatol. 2017;29(4):481-7. doi: 10.1097/MEG.0000000000000820. [PubMed: 28253211].

12. Agopian VG, Kaldas FM, Hong JC, Whittaker M, Holt C, Rana $A$, et al. Liver transplantation for nonalcoholic steatohepatitis: the new epidemic. Ann Surg. 2012;256(4):624-33. doi: 10.1097/SLA.0b013e31826b4b7e. [PubMed: 22964732].

13. Bhagat V, Mindikoglu AL, Nudo CG, Schiff ER, Tzakis A, Regev A. Outcomes of liver transplantation in patients with cirrhosis due to nonalcoholic steatohepatitis versus patients with cirrhosis due to alcoholic liver disease. Liver Transpl. 2009;15(12):1814-20. doi: 10.1002/lt.21927. [PubMed: 19938128].

14. Reddy SK, Steel JL, Chen HW, DeMateo DJ, Cardinal J, Behari J, et al. Outcomes of curative treatment for hepatocellular cancer in nonalcoholic steatohepatitis versus hepatitis $\mathrm{C}$ and alcoholic liver disease.Hepatology.2012;55(6):1809-19. doi:10.1002/hep.25536. [PubMed: 22183968].

15. Vanwagner LB, Bhave M, Te HS, Feinglass J, Alvarez L, Rinella ME. Patients transplanted for nonalcoholic steatohepatitis are at increased risk for postoperative cardiovascular events. Hepatology. 2012;56(5):1741-50. doi: 10.1002/hep.25855. [PubMed: 22611040].

16. Wang X, Li J, Riaz DR, Shi G, Liu C, Dai Y. Outcomes of liver transplantation for nonalcoholic steatohepatitis: A systematic review and meta-analysis. Clin Gastroenterol Hepatol. 2014;12(3):394-402 e1. doi 10.1016/j.cgh.2013.09.023. [PubMed: 24076414].

17. Singal AK, Guturu P, Hmoud B, Kuo YF, Salameh H, Wiesner RH. Evolving frequency and outcomes of liver transplantation based on etiology of liver disease. Transplantation. 2013;95(5):755-60. doi 10.1097/TP.ob013e31827afb3a. [PubMed: 23370710].

18. Afzali A, Berry K, Ioannou GN. Excellent posttransplant survival for patients with nonalcoholic steatohepatitis in the United States. Liver Transpl. 2012;18(1):29-37. doi: 10.1002/lt.22435. [PubMed: 21932374].

19. Charlton MR, Burns JM, Pedersen RA, Watt KD, Heimbach JK, Dierkhising RA. Frequency and outcomes of liver transplantation for nonalcoholic steatohepatitis in the United States. Gastroenterology. 2011;141(4):1249-53. doi: 10.1053/j.gastro.2011.06.061. [PubMed: 21726509].

20. Contos MJ, Cales W, Sterling RK, Luketic VA, Shiffman ML, Mills AS et al. Development of nonalcoholic fatty liver disease after orthotopic liver transplantation for cryptogenic cirrhosis. Liver Transpl. 2001;7(4):363-73. doi: 10.1053/jlts.2001.23011. [PubMed: 11303298].

21. Montori VM, Basu A, Erwin PJ, Velosa JA, Gabriel SE, Kudva YC. Posttransplantation diabetes: A systematic review of the literature. Diabetes Care. 2002;25(3):583-92. doi: 10.2337/diacare.25.3.583. [PubMed: $11874952]$.

22. Lorenz MW, Markus HS, Bots ML, Rosvall M, Sitzer M. Prediction of clinical cardiovascular events with carotid intima-media thickness: A systematic review and meta-analysis. Circulation. 2007;115(4):459-67. doi: 10.1161/CIRCULATIONAHA.106.628875. [PubMed: 17242284].

23. Lease ED. Infections and sepsis after liver transplantation. In: Doria C, editor. Contemporary liver transplantation. Springer, Cham; 2015. p. 1-13. doi:10.1007/978-3-319-05543-5_19-1.

24. Van den Berg EH, Douwes RM, de Meijer VE, Schreuder T, Blokzijl H Liver transplantation for NASH cirrhosis is not performed at the expense of major post-operative morbidity. Dig Liver Dis. 2018;50(1):6875. doi: 10.1016/j.dld.2017.08.022. [PubMed: 28935188] 